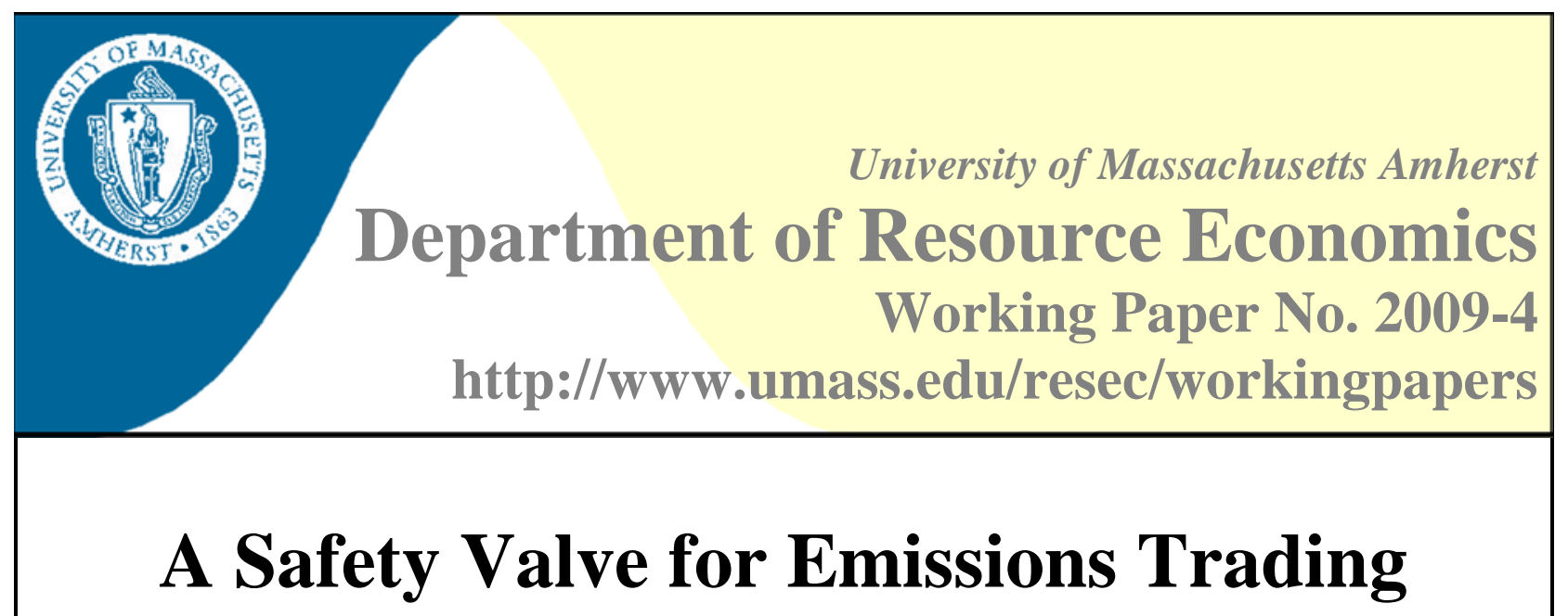

\author{
John K. Stranlund ${ }^{1}$
}

Abstract:

This paper considers the optimal design of an emissions trading program that includes a safety valve tax that allows pollution sources to escape the emissions cap imposed by the aggregate supply of emissions permits. I demonstrate that an optimal hybrid emissions trading/emissions tax policy involves a permit supply that is strictly less than under a pure emissions trading scheme and a safety valve tax that exceeds the optimal pure emissions tax as long as expected marginal damage is an increasing function. While the expected level of emissions under a hybrid policy may be more or less than under pure emissions trading or a pure emissions tax, under the assumption that uncertainty about aggregate marginal abatement costs is symmetric the most likely outcome is that emissions will turn out to be less under the hybrid. Finally, a steeper expected marginal damage function calls for higher permit supply and safety valve, which reduces expected aggregate emissions and the probability that the safety valve will be employed.

Keywords: Emissions Taxes, Emissions Trading, Uncertainty, Safety Valve, Hybrid Emissions Control

JEL Classification: L51, Q28

\footnotetext{
${ }^{1}$ John K. Stranlund, Department of Resource Economics

University of Massachusetts, 214 Stockbridge Hall

80 Campus Center Way, Amherst, MA 01003-9246

E: stranlund@resecon.umass.edu P: 413-545-6328 F: 413-545-5853
} 
July 2009

\title{
A Safety Valve for Emissions Trading
}

\author{
JOHN K. STRANLUND \\ Department of Resource Economics \\ University of Massachusetts-Amherst
}

\begin{abstract}
This paper considers the optimal design of an emissions trading program that includes a safety valve tax that allows pollution sources to escape the emissions cap imposed by the aggregate supply of emissions permits. I demonstrate that an optimal hybrid emissions trading/emissions tax policy involves a permit supply that is strictly less than under a pure emissions trading scheme and a safety valve tax that exceeds the optimal pure emissions tax as long as expected marginal damage is an increasing function. While the expected level of emissions under a hybrid policy may be more or less than under pure emissions trading or a pure emissions tax, under the assumption that uncertainty about aggregate marginal abatement costs is symmetric the most likely outcome is that emissions will turn out to be less under the hybrid. Finally, a steeper expected marginal damage function calls for higher permit supply and safety valve, which reduces expected aggregate emissions and the probability that the safety valve will be employed.
\end{abstract}

Keywords: Emissions Taxes, Emissions Trading, Uncertainty, Safety Valve, Hybrid Emissions Control

JEL Codes: L51, Q28.

Correspondence to: John K. Stranlund, Department of Resource Economics, 214 Stockbridge Hall, 80 Campus Center Way, University of Massachusetts-Amherst, Amherst, MA 01003, USA. Phone: (413) 545-6328, Fax: (413) 545-5853, E-mail: stranlund@resecon.umass.edu. 


\section{A Safety Valve for Emissions Trading}

\section{Introduction}

Much attention has been devoted recently to adding safety valves to cap-and-trade policies. A safety valve is an emissions tax that allows sources of pollution to emit beyond on their emissions permits. ${ }^{1}$ A safety valve tax effectively places a price ceiling on emissions permits, thus reducing uncertainty about marginal abatement costs. The theoretical motivation for hybrid emissions trading/emissions tax policies under uncertainty about the benefits and costs of pollution control originated with Roberts and Spence (1976). They showed that since a pure emissions tax and pure emissions trading are special cases of a hybrid scheme, an optimal combination of taxes and emissions trading cannot be less efficient than either a pure tax or a pure trading scheme. In most cases a hybrid will outperform both pure schemes.

The current debate about safety valves has been driven by their proposed use in cap-andtrade climate change policies to contain the highly uncertain costs of controlling greenhouse gases (Jacoby and Ellerman 2004). ${ }^{2}$ Some climate policy proposals have safety valves that are so low that there would be a high likelihood that they would be used (e.g., \$10 per ton of CO2 in the proposal of McKibbin and Wilcoxen (1997, 2002); \$7 per metric ton of CO2-equivalent rising at 5\% per year in Holdren et al. 2004). On the other hand, Stavins (2008) proposes using a safety valve that is so high that it would have little chance of being employed. ${ }^{3}$

\footnotetext{
${ }^{1}$ An equivalent interpretation is that a safety valve is a price at which the government commits to selling an unlimited number of emission permits.

${ }^{2}$ Samuelsohn (2008) provides a very brief recent history of the debate about proposals for safety valves in U.S. climate policy.

${ }^{3}$ See Aldy et al. (2003) for several other climate policy proposals that are permit trading/safety valve hybrids. Some view the relatively high penalties for permit violations in the U.S. $\mathrm{SO}_{2}$ Allowance Trading and the E.U. Emissions Trading Scheme as safety valves because they place
} 
Most authors who propose or comment on safety valves for emissions trading do not determine economically efficient hybrid policies. ${ }^{4}$ The sole exception may be Pizer (2002), who developed a simulation model to determine the optimal permit supply and safety valve for confronting climate change. Pizer's (2002) calculations suggest that the optimal hybrid policy in a particular year is to set a safety valve that is equal to the optimal pure tax, while supplying significantly fewer permits than under pure emissions trading. Under this program there would be a high probability that the safety valve is used and expected aggregate emissions would exceed the level that equates expected marginal damage and marginal abatement costs. An important characteristic of the control greenhouse gases is that the expected marginal damage function in a particular year is estimated to be almost perfectly flat. ${ }^{5}$

However, there appears to be no published work that tells us whether Pizer's results hold beyond controlling greenhouse gases. In this paper I examine the optimal design of an emissions trading program with a safety valve using an analytical model of linear aggregate marginal abatement costs and marginal damage with uncertainty about the intercepts of these functions. ${ }^{6}$

a ceiling on the price of emissions permits in these programs (Jacoby and Ellerman 2004). These penalties are so high relative to allowance prices that they are rarely applied.

${ }^{4}$ To be fair, some like Stavins (2008) are as concerned about political realities that favor emissions trading over emissions taxes as they are about economic efficiency.

${ }^{5}$ This is true mainly because greenhouse gases are stock pollutants and their emissions in any one year do not add much to the accumulated stock.

${ }^{6}$ Other authors have proposed hybrid schemes that are more complicated than the basic trading/tax scheme that is usually proposed. In fact, Roberts' and Spence's main proposal also has a price floor to subsidize emissions reductions below the permit supply. Burtraw et al. (2009) and Philibert (2008) examine price ceilings and floors in simulation studies of greenhouse gas control but neither attempt to determine optimal floors and ceilings. In part to assuage environmental advocates' fears of an unlimited quantity of carbon emissions available at a safety valve, Murray et al. (2009) propose an "allowance reserve" which limits how far emissions can exceed the supply of permits at the safety valve. Each of these proposals have merit, but I chose to limit this study to determining an optimal trading scheme with a simple safety valve, because these policies are proposed most often. 
This model has been used extensively to examine optimal pollution control under uncertainty since Weitzman's (1974) seminal work on price vs. quantity controls. I first demonstrate that the optimal supply of permits under a hybrid policy is always less than the permit supply under pure emissions trading. Moreover, the safety valve is exactly equal to the optimal pure tax when marginal damage is a constant. However, the optimal safety valve is strictly greater than the pure tax when marginal damage is increasing. Thus, Pizer's result that the optimal safety valve is equal to the optimal pure tax follows because expected marginal damage from greenhouse gas emissions in a particular year is essentially constant. The safety valve is higher when expected marginal damage is increasing to limit the potential damage associated with allowing pollution sources to escape the permit supply.

Given results about the optimal permit supply and safety valve in a hybrid trading/tax policy, it is important to ask whether a hybrid policy produces more or less emissions control (in expectation) than pure emissions trading or a pure emissions tax. Of course, expected emissions under a pure tax are equal to the permit supply under pure emissions trading; both are equal to the level of emissions that equates expected marginal damage and expected marginal abatement costs. I demonstrate that the relationship between expected emissions under a hybrid policy and expected emissions under pure emissions trading or a pure emissions tax is indeterminate when marginal damage is increasing. The reason for this indeterminacy is that while emissions are strictly less than the level that equates expected marginal damage and marginal abatement cost when the safety valve is not used (because of the low permit supply), expected emissions when the safety valve is used are strictly greater than that level. However, under the assumption that the uncertainty in the intercept of the aggregate marginal abatement cost function is symmetrically distributed, the probability that the ex post level of emissions is greater under a 
hybrid policy is strictly less than one-half. Thus, while expected emissions under a hybrid policy may be greater or less than under pure emissions trading or a pure tax, the most likely outcome is that emissions will turn out to be less under the hybrid.

One of the main results of this work is that that the slope of the expected marginal damage function plays a fundamental role in forming a hybrid policy. I show that a steeper expected marginal damage function calls for both a higher permit supply and safety valve. These two effects decrease the probability the safety valve will be used and reduce expected aggregate emissions. Thus, a steeper expected marginal damage function implies that the value of controlling abatement cost uncertainty with a safety valve is reduced relative to the value of controlling aggregate emissions uncertainty with the supply of permits

\section{An optimal emissions trading/emissions tax hybrid}

Suppose that the aggregate emissions of a uniformly mixed pollutant is $E$. Suppose further that emissions of $E$ are controlled by a cost-effective instrument, like a competitive emissions trading scheme, an emissions tax, or some combination. Let the minimum aggregate abatement cost function of the sources of $E$ be $C(E, u)$, where $u$ is a random variable. Let us suppose that $C(E, u)=C(E)-u E$, so that uncertainty about abatement costs is uncertainty about the intercept of the aggregate marginal abatement cost function; that is,

$$
-\partial C(E, u) / \partial E=-C^{\prime}(E)+u .
$$

Suppose throughout that $u$ has a probability density function $f(u)$, support $[\underline{u}, \bar{u}]$, and zero expectation. The latter assumption implies that $-C^{\prime}(E)$ is the expected aggregate marginal abatement cost function. 
Since the pollutant is uniformly mixed, damage from pollution depends on aggregate emissions, not the geographical distribution of emissions. Let the expected damage function be $D(E)+\delta E$, where $\delta$ is a random variable with zero mean and $D(E)$ is strictly increasing and convex (perhaps weakly convex). Assume that uncertainty about the damage function is uncorrelated with uncertainty about abatement costs. This implies the well-known result that uncertainty about pollution damage is irrelevant to the choice of an optimal control policy. Thus, in our analysis we need only be concerned about expected damage $D(E)$.

Let $L$ and $t$ be the aggregate supply of permits and the safety valve tax, respectively, under a hybrid emissions trading/emissions tax scheme. Which policy variable is the binding one depends on the realization of aggregate marginal abatement costs; that is, on the realization of the random parameter $u$. If aggregate marginal abatement costs turn out to be low enough so that $-C^{\prime}(L)+u \leq t$, then aggregate emissions are limited to the supply of permits and their price is $p=-C^{\prime}(L)+u$. However, if aggregate marginal abatement costs are high enough so that $-C^{\prime}(L)+u>t$, then the price of permits will rise to the level of the safety valve. Firms will take advantage of the fixed emissions price by increasing their emissions beyond the supply of permits to

$$
E(t, u)=E \mid-C^{\prime}(E)+u=t .
$$

Note that we can define a cut-off value of the random variable $u$ at which aggregate emissions are equal to the permit supply and the price of permits is equal to the safety valve. This value of $u$ is

$$
u^{0}=u^{0}(t, L)=t+C^{\prime}(L) .
$$

At $u=u^{0}$ [2] and [3] imply

$$
E\left(t, u^{0}\right)=L .
$$


Let $W(L, t)$ denote the expected social costs of pollution and its control under a hybrid trading/tax policy $(L, t) . W(L, t)$ is the sum of expected aggregate abatement costs plus expected pollution damage. To choose an optimal hybrid policy the regulator chooses $L$ and $t$ to minimize:

$W(L, t)=\int_{\underline{u}}^{u^{0}}[C(L)-u L+D(L)] f(u) d u+\int_{u^{0}}^{\bar{u}}[C(E(t, u))-u E(t, u)+D(E(t, u))] f(u) d u$

s.t. $u^{0}=t+C^{\prime}(L)$.

A pure emissions trading scheme and a pure emissions tax are possible solutions to [5]. In these cases we would find $u^{0} \geq \bar{u}$ for pure emissions trading, and $u^{0} \leq \bar{u}$ for a pure emissions tax. In this paper, however, let us focus on strict hybrid policies, that is, those policies that involve strictly positive probabilities that aggregate emissions will be limited by the permit supply $L$ and that the permit price will be limited by the safety valve $t$. Thus, we constrain the solution to [5] by $u^{0} \in(\underline{u}, \bar{u})$.

Moreover, assume that $W(L, t)$ is strictly convex and that the optimal policy calls for $L>0$ and $t>0$. Then, the following first-order conditions are both necessary and sufficient to determine an optimal hybrid emissions control policy:

$$
\begin{aligned}
W_{L}(L, t) & =\int_{\underline{u}}^{u^{0}}\left[C^{\prime}(L)-u+D^{\prime}(L)\right] f(u) d u \frac{\partial u^{0}}{\partial L}\left[C(L)-u^{0} L+D(L)\right] f\left(u^{0}\right) \\
& -\frac{\partial u^{0}}{\partial L}\left[C\left(E\left(t, u^{0}\right)\right)-u^{0} E\left(t, u^{0}\right)+D\left(E\left(t, u^{0}\right)\right)\right] f\left(u^{0}\right)=0 ; \\
W_{t}(L, \pi) & =\int_{u^{0}}^{\bar{u}}\left\{\left[C^{\prime}(E(t, u))-u+D^{\prime}(E(t, u))\right] E_{t}(t, u)\right\} f(u) d u+\frac{\partial u^{0}}{\partial t}\left[C(L)-u^{0} L+D(L)\right] f\left(u^{0}\right) \\
& -\frac{\partial u^{0}}{\partial t}\left[C\left(E\left(t, u^{0}\right)\right)-u^{0} E\left(t, u^{0}\right)+D\left(E\left(t, u^{0}\right)\right)\right] f\left(u^{0}\right)=0 .
\end{aligned}
$$

These conditions can be simplified substantially. Since $E\left(t, u^{0}\right)=L$ (from [4]) the second and third terms of both [6] and [7] sum to zero. Furthermore, since $E(t, u)$ is the implicit solution to 
$-C^{\prime}(E)+u=t,-C^{\prime}(E(t, u))+u \equiv t$ and $E_{t}(t, u)=-1 / C^{\prime \prime}(E)<0$. Ultimately, [6] and [7] can be written as:

$$
\begin{aligned}
& W_{L}(L, t)=\int_{\underline{u}}^{u^{0}}\left[C^{\prime}(L)-u+D^{\prime}(L)\right] f(u) d u=0 ; \\
& W_{t}(L, t)=\frac{1}{C^{\prime \prime}} \int_{u^{0}}^{\bar{u}}\left[t-D^{\prime}(E(t, u))\right] f(u) d u=0 .
\end{aligned}
$$

Most the analysis of the paper relies on a common linearization of the model. Let

$$
-C^{\prime}(E)+u=c-C^{\prime \prime} E+u
$$

be aggregate marginal abatement costs, and let

$$
D^{\prime}(E)=d+D^{\prime \prime} E
$$

be expected marginal damage. Using [10] and the definition of $E(t, u)$ provided by [2],

$$
E(t, u)=\frac{c-t+u}{C^{\prime \prime}}
$$

Our assumption that there is a positive probability that pollution sources will employ the safety valve implies that $E(t, u)>0$ throughout the paper.

Having provided all the ingredients of the model, the main results of this section are qualitative comparisons of the permit supply under the hybrid policy to the permit supply under pure emissions trading and the safety valve under a hybrid to a pure emissions tax. That is, we will compare the permit supply and safety valve to the permit supply and tax that equate expected marginal abatement costs and expected marginal damage. Using [2], [10], and [11], these values are:

$$
\begin{aligned}
& \hat{E}=(c-d) /\left(C^{\prime \prime}+D^{\prime \prime}\right) \\
& \hat{t}=\left(d C^{\prime \prime}+c D^{\prime \prime}\right) /\left(C^{\prime \prime}+D^{\prime \prime}\right) .
\end{aligned}
$$


Our first result compares the supply of permits under a hybrid policy to $\hat{E}$ and gives it a lower bound. The proofs of all propositions are in the Appendix.

Proposition 1: Under an emissions trading/emissions tax hybrid, the optimal supply of permits is strictly less than the supply of permits that equates expected marginal abatement costs and expected marginal damage. Moreover, the optimal permit supply is strictly greater than the level of emissions that equates expected marginal damage and the lowest possible marginal abatement cost function.

Proposition 2 compares the optimal safety valve to $\hat{t}$ and specifies its upper bound.

Proposition 2: Under an emissions trading/emissions tax hybrid, the safety valve exceeds the price that equates expected marginal damage and expected marginal abatement costs if and only if the expected marginal damage function is strictly increasing. The safety valve is equal to the price that equates marginal damage and expected marginal abatement costs if marginal damage is constant. Finally, when marginal damage is increasing the safety valve is strictly less than the tax that equates expected marginal damage and the highest possible marginal abatement cost function.

The proof of Proposition 1 indicates that the results do not depend on linearity in marginal damage and marginal abatement costs. The result in Proposition 2 that $\hat{t}$ is the optimal safety valve when expected marginal damage is constant also does not require linearity of the marginal abatement cost and marginal damage functions. Simply observe in [9] that if 
$D^{\prime}(E(t, u))$ is a positive constant $D^{\prime}$, then $t=D^{\prime}$ is required for $W_{t}(L, t)=0$. Therefore, these results are consistent with Pizer’s (2002) simulation results of an optimal hybrid policy for greenhouse gases. Recall that he also found a reduced permit supply and a safety valve equal to the close-to-constant expected marginal damage function he estimated.

However, Propositions 1 and 2 also offer characteristics of hybrid policies for pollutants that cause increasing marginal damage. In these cases, it is still true that the permit supply is lower than under a pure emissions trading policy. However, the safety valve is higher than under a pure emissions tax. This higher safety valve serves to limit the potential damage that can arise from allowing pollution sources to emit beyond the permit supply. We will return several times throughout the analysis to the role the slope of the expected marginal damage function plays in determining optimal hybrid policies.

Propositions 1 and 2 also place lower and upper bounds on the optimal permit supply and optimal safety valve, respectively. We will use these bounds when we construct a graph of the optimal hybrid policy in a later section of the paper.

\section{Does a hybrid scheme promote more or less environmental control?}

Having characterized an optimal hybrid policy, we can now address whether such a policy produces more or less emissions control (in expectation) than a pure trading policy or a pure emissions tax. Recall that expected aggregate emissions under the tax and certain aggregate emissions under a pure trading scheme are both equal to $\hat{E}$. The question of whether a hybrid scheme promotes more control is answered by the sign of

$$
L \int_{\underline{u}}^{u^{0}} f(u) d u+\int_{u^{0}}^{\bar{u}} E(t, u) f(u) d u-\hat{E} .
$$


The first two terms of [15] provide the expectation of aggregate emissions under a hybrid scheme. Noting that

$$
\hat{E}=\hat{E} \int_{\bar{u}}^{u^{0}} f(u) d u+\hat{E} \int_{\bar{u}}^{u^{0}} f(u) d u,
$$

[15] can be rewritten as

$$
(L-\hat{E}) \int_{\bar{u}}^{u^{0}} f(u) d u+\left\{\frac{\int_{u^{0}}^{\bar{u}} E(t, u) f(u) d u}{\int_{u^{0}}^{\bar{u}} f(u) d u}-\hat{E}\right\} \int_{u^{0}}^{\bar{u}} f(u) d u .
$$

The first term of [16] is the difference between the permit supply and $\hat{E}$ times the probability that the safety valve will not be used (and aggregate emissions are equal to the permit supply). We know from Proposition 2 that $L<\hat{E}$ so the first term of [16] is negative. The second term of [16] is the difference between the conditional expectation of emissions, given that the safety valve will be used, and $\hat{E}$ all times the probability that the safety valve will be used. If expected emissions when the safety valve is used is less than or equal to $\hat{E}$, then [16] would be negative, indicating that expected emissions under the hybrid policy would be strictly less than $\hat{E}$. However, the following proposition indicates that expected emissions when the safety valve is used are strictly greater than $\hat{E}$, leaving the sign of [16] indeterminate.

Proposition 3: Expected aggregate emissions conditional on firms using the safety valve are strictly greater than the level of emissions that equates marginal damage and expected marginal abatement costs. 
Propositions 1 and 3 indicate why expected aggregate emissions under a hybrid scheme can be greater than, less than, or equal to emissions under a pure trading scheme and expected emissions under a pure emissions tax. When the permit price is less than or equal to the safety valve aggregate emissions are held to the permit supply, which is strictly less than $\hat{E}$. However, expected emissions exceed $\hat{E}$ when firms use the safety valve. Whether a hybrid scheme will produce more or less emissions control than pure trading or tax polices depends in part on these differences.

But it is also clear from [19] that the relative level of control of a hybrid policy depends on the probabilities that the safety valve is and is not employed. Under the assumption that uncertainty about the marginal abatement cost function is distributed symmetrically, the following proposition reveals that these probabilities depend on the relative slopes of the expected marginal damage and expected marginal abatement cost functions.

Proposition 4: Suppose that that the distribution of $u$ is symmetric. Under an emissions trading/emissions tax hybrid the probability that the safety valve will be employed is less than one-half if and only if $D^{\prime \prime}>C^{\prime \prime}$.

Proposition 4 is related to Weitzman's (1974) result about the relative efficiency of price and quantity controls. Restricted to pure tax or pure trading policies, emissions trading is preferred if the expected marginal damage function is steeper than the expected marginal abatement cost function. The reason is that it is more efficient to cap emissions than to cap marginal abatement costs in this case. Under a hybrid scheme when the expected marginal damage function is relatively steeper, the preference for the emissions cap translates into a 
relatively low probability that the cap will be exceeded (i.e., that the safety valve is used). However, when the expected marginal abatement cost function is steeper than the marginal damage function, a pure tax is preferred to pure emissions trading. A hybrid policy in this case will reflect this preference for a tax by making the use of the safety valve more likely than not.

One next result gives us a clearer picture of the level of control under a hybrid scheme. It indicates that while expected emissions under a hybrid policy may be greater or less than the level that equates expected marginal damage and expected marginal abatement cost, the most likely outcome is that emissions will turn out to be less than this value.

Proposition 5: Suppose that the distribution of $u$ is symmetric. Under an emissions trading/ emissions tax hybrid, the probability that aggregate emissions are greater than the level that equates expected marginal abatement cost and expected marginal damage is strictly less than one-half if marginal damage is increasing. The probability that emissions exceed the level that equates expected marginal abatement cost and expected marginal damage is equal to one-half if expected marginal damage is a constant.

To complete the analysis let us examine the role of the slope of the expected marginal damage on the optimal hybrid policy more thoroughly. We conduct a comparative static analysis with respect to $D^{\prime \prime}$, but take a somewhat different approach than is conventional. Note that increasing $D^{\prime \prime}$ without changing the intercept of the expected marginal damage function both increases its steepness and shifts it up. To focus solely on the effect of the steepness of the expected marginal damage function; that is, to avoid the confounding effect of shifting the marginal damage function up, let us change the slope of the function and its intercept 
simultaneously so that it rotates around $(\hat{E}, \hat{t})$. That is, let us model $D^{\prime}(E)=d+D^{\prime \prime} E$ with $d$ a function of $D^{\prime \prime}$, and choose $d\left(D^{\prime \prime}\right)$ so that changing $D^{\prime \prime}$ maintains $(\hat{E}, \hat{t})$ as the intersection of expected marginal damage and expected marginal abatement costs. This comparative static exercise produces the following results.

Proposition 6: Suppose that the expected marginal damage function is upward sloping. Consider changing this function by rotating it around $(\hat{E}, \hat{t})$. Making the expected marginal damage function steeper (shallower) in this way:

1) Increases (decreases) both the supply of permits and the safety valve of an optimal hybrid control policy.

2) Decreases (increases) the probability that the safety valve will be used.

3) Decreases (increases) expected emissions.

When the expected marginal damage function is steeper the optimal hybrid policy limits the ability of firms to escape the cap imposed by the supply of permits. This is accomplished by increasing both the supply of permits and increasing the safety valve. Both actions reduce the probability that the safety valve will be employed, which reflects the notion that it becomes relatively more important to limit aggregate emissions than marginal abatement costs when marginal damage is steeper. Moreover, a steeper expected marginal damage function results in lower expected emissions. This effect has three components. The first is the increase in the permit supply, which will actually tend to increase expected emissions. However, the lower probability that the safety valve will be used tends to reduce expected emissions. Finally the higher safety valve reduces the expectation of aggregate emissions given the safety valve is 
employed. The first effect is dominated by the latter two effects so that expected emissions are lower when the expected marginal damage function is steeper. ${ }^{7}$

\section{Graphical summary of the main results.}

We can summarize the main results of this work with simple graphs. Figure 1 is a graph of a hybrid policy for an increasing expected marginal damage function and a symmetric distribution of the random variable $u$. The optimal supply of emissions permits under a pure emissions trading program $\hat{E}$ and the optimal pure emissions tax $\hat{t}$ equate expected marginal abatement costs $-C^{\prime}(E)$ and expected marginal damage $D^{\prime}(E)$. The optimal hybrid policy is illustrated by the bold step function. From Proposition 1, the supply of permits under a hybrid scheme is strictly less than $\hat{E}$ but strictly greater than the level of emissions that equates expected marginal damage and the lowest possible marginal abatement cost function, $-C^{\prime}(E)+\underline{u}$. From Proposition 2, an increasing expected marginal damage function makes the safety valve for a hybrid policy strictly greater than $\hat{t}$, but strictly less than the tax that would equate expected marginal damage and the highest possible marginal abatement cost function, $-C^{\prime}(E)+\bar{u}$.

In Figure 1 the expected marginal damage function is not as steeply sloped as the expected marginal abatement cost function. From Proposition 4 this implies that $u^{0}<0$ and the probability the safety valve will be used is strictly greater than one-half. What cannot be seen in the graph is the result that expected emissions conditional on the safety valve being used are

\footnotetext{
${ }^{7}$ I have conducted this same analysis for the steepness of the expected marginal abatement cost function. The effects of rotating the expected marginal abatement cost function around $(\hat{E}, \hat{t})$ on the permit supply, the safety valve, the probability of using the safety valve, and expected emissions are all ambiguous.
} 
strictly greater than $\hat{E}$ (Proposition 3), and the probability that expected emissions from the hybrid policy exceed $\hat{E}$ is less than one-half (Proposition 5).

To illustrate the results of Proposition 6, Figure 2 includes a steeper expected marginal damage function, $D_{S}^{\prime}(E)$, that is rotated counter clockwise around $(\hat{E}, \hat{t})$. From Proposition 6, the supply of permits and the safety valve increase to $L_{S}$ and $t_{S}$, respectively. $D_{S}^{\prime}(E)$ is drawn so that it is steeper than the expected marginal abatement cost function. From Proposition 4, $L$ and $t$ are set so that $u_{S}^{0}>0$, which implies that the probability the safety valve will be used is less than one-half if the distribution of $u$ is symmetric. Since $u^{0}$ increases as the expected marginal damage function get steeper, the probability that the safety valve is used falls. The second part of Proposition 6 indicates that the probability the safety valve is used falls as the expected marginal damage curve gets steeper. In addition, expected aggregate emissions decrease as expected marginal damage gets steeper.

\section{Conclusion}

In this paper I have analyzed the design of an optimal hybrid emissions trading/emissions tax pollution control policy. A hybrid policy improves efficiency by trading off uncertainty in aggregate emissions against uncertainty in the price of emissions. Under a pure tax there is no uncertainty about the emissions price, but ex post aggregate emissions can be highly uncertain if the aggregate marginal abatement cost function is highly uncertain. On the other hand, a pure trading scheme eliminates uncertainty about aggregate emissions (if well enforced, aggregate emissions are equal to the supply of permits), but the price of emissions can be highly uncertain. A hybrid trading/tax policy improves upon a pure tax by allowing some price uncertainty to 
reduce emissions uncertainty: a hybrid policy improves upon pure emissions trading by allowing some emissions uncertainty to reduce price uncertainty.

This tradeoff is particular sensitive to the slope of the marginal damage function. As this function gets steeper it becomes relatively more important to control emissions uncertainty than price uncertainty. This is accomplished by increasing both the supply of permits and the safety valve tax. These two actions decrease the probability the safety valve will be used and reduce expected aggregate emissions.

There are several ways to extend the analysis of this paper, but let me conclude with one extension that I think is the most important. Since the analysis of this paper is static, extending the determination of hybrid policies into dynamic environments would be useful, particularly when emissions trading includes provisions for banking and borrowing emissions permits. In fact, permit banking and borrowing can serve to dampen permit price fluctuations like a safety valve. Jacoby and Ellerman (2004) suggest that a safety valve will be particular useful in initial periods of an emissions trading program before a permit bank has been built up and when uncertainty about abatement costs will be greatest. In the longer run, however, giving pollution sources the ability to bank permits and to borrow from future allocations can help contain abatement cost uncertainty like a safety valve does. The optimal determination of a safety valve combined with banking and borrowing provisions is likely to be a worthwhile exercise. 


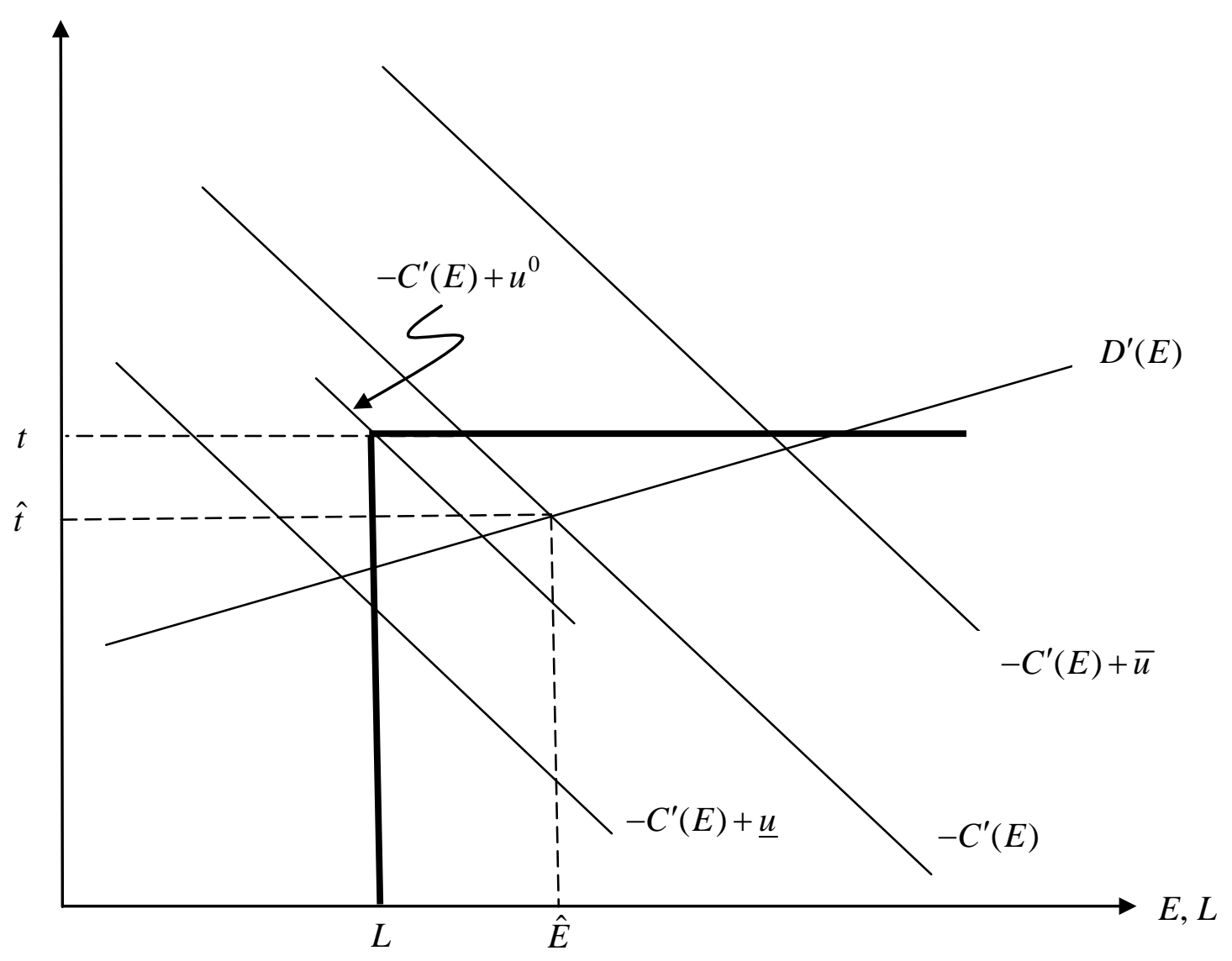

Figure 1: An optimal hybrid policy $(L, t)$. The optimal supply of emissions permits under a pure emissions trading program is $\hat{E}$. The optimal pure emissions tax is $\hat{t} . L<\hat{E}$ from Proposition 1 and $t>\hat{t}$ from Proposition 2. The graph is drawn so that the expected marginal damage function is not as steep as the expected marginal abatement cost function. In these situations, Proposition 4 indicates that $(L, t)$ are set so that $u^{0}<0$. This implies that the probability the safety valve will be used is greater than $1 / 2$ if the distribution of $u$ is symmetric around its zero mean. 


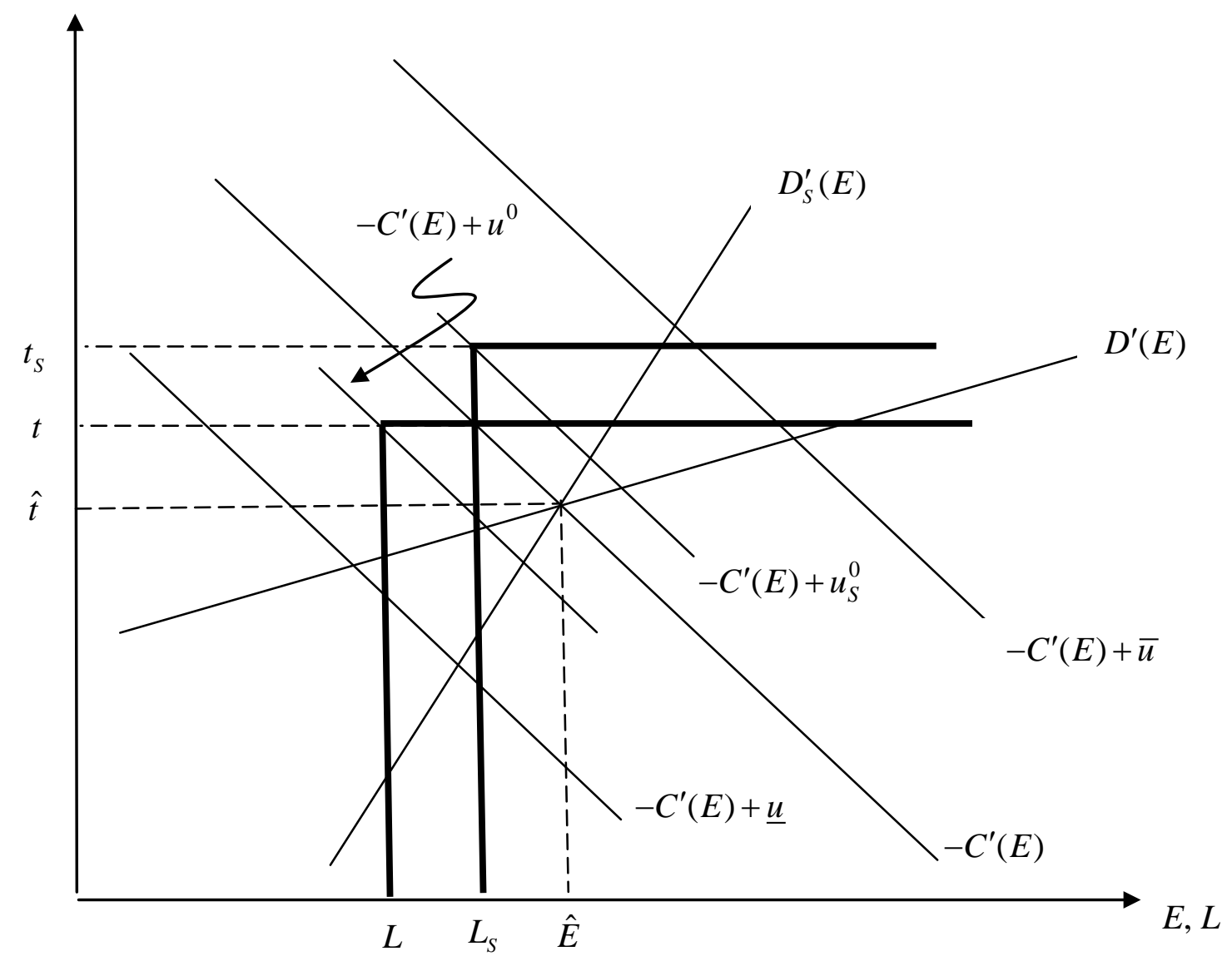

Figure 2: If the expected marginal damage function is steeper, say $D_{s}^{\prime}(E)$, Proposition 6 indicates that the supply of permits and the safety valve increase to $L_{S}$ and $t_{S}$, respectively. This increases $u^{0}$ to $u_{s}^{0}$, which indicates that the probability the safety valve is used decreases. This illustrates the second part of Proposition 6. In fact, $D_{S}^{\prime}(E)$ is drawn so that the expected marginal damage function is steeper than the expected marginal abatement cost function. Proposition 4 indicates that $(L, t)$ should be set so that $u_{S}^{0}>0$, indicating the probability the safety valve will be used is less than $1 / 2$ if the distribution of $u$ is symmetric. 


\section{Appendix}

Proof of Proposition 1: Rewrite [8] as

$$
W_{L}(L, t)=\left[C^{\prime}(L)+D^{\prime}(L)\right] \int_{\underline{u}}^{u^{0}} f(u) d u-\int_{\underline{u}}^{u^{0}} u f(u) d u=0
$$

Under a hybrid policy, $u^{0}<\bar{u}$. Since the expectation of $u$ is equal to zero, $u^{0}<\bar{u}$ implies

$\int_{\underline{u}}^{u^{0}} u f(u) d u<0$. Moreover, $\int_{\underline{u}}^{u^{0}} f(u) d u>0$, because this is the probability that $u \leq u^{0}$. Using

[A.1], let us now evaluate the sign of $W_{L}(L, t)$ at $L=\hat{E}$. Since $\hat{E}$ is the solution to $C^{\prime}(E)+D^{\prime}(E)=0$

$$
W_{L}(L=\hat{E}, t)=-\int_{\underline{u}}^{u^{0}} u f(u) d u>0 .
$$

Since $W$ is strictly convex, $W_{L}(L=\hat{E}, t)>0$ implies that the optimal supply of permits is strictly less than $\hat{E}$.

To prove the second part of Proposition1, first note that the lowest possible marginal abatement cost function is $-C^{\prime}(E)+\underline{u}$. Let $\bar{E}$ be the solution to $-C^{\prime}(E)+\underline{u}=D(E)$. Using [A.1], we now evaluate the sign of $W_{L}(L, t)$ at $L=\bar{E}$ :

$W_{L}(L=\bar{E}, t)=\left[C^{\prime}(\bar{E})+D^{\prime}(\bar{E})\right] \int_{\underline{u}}^{u^{0}} f(u) d u-\int_{\underline{u}}^{u^{0}} u f(u) d u=\int_{\underline{u}}^{u^{0}} \underline{u} f(u) d u-\int_{\underline{u}}^{u^{0}} u f(u) d u<0$.

The sign of $W_{L}(L=\bar{E}, t)$ follows because $\underline{u}$ is the lowest possible $u$. Since $W$ is strictly convex, $W_{L}(L=\bar{E}, t)<0$ implies that the optimal permit supply is strictly greater than $\bar{E}$. QED

Proof of Proposition 2: Begin by substituting [12] into [11] to obtain 


$$
D^{\prime}(E(t, u))=d+\frac{D^{\prime \prime}(c-t)}{C^{\prime \prime}}+\frac{D^{\prime \prime} u}{C^{\prime \prime}}
$$

Note that [A.2] can be written as

$$
D^{\prime}(E(t, u))=D^{\prime}(E(t, u=0))+\frac{D^{\prime \prime} u}{C^{\prime \prime}}
$$

Substitute this into [9] to obtain

$$
W_{t}(L, t)=\frac{1}{C^{\prime \prime}} \int_{u^{0}}^{\bar{u}}\left[t-D^{\prime}(E(t, u=0))-\frac{D^{\prime \prime} u}{C^{\prime \prime}}\right] f(u) d u=0
$$

Let us now evaluate the sign of $W_{t}(L, t)$ at $t=\hat{t}$. Since $\hat{t}$ equates expected marginal damage and expected marginal abatement cost, firms' expected response to $\hat{t}$ is $E(\hat{t}, u=0)$ and, consequently, $\hat{t}=D^{\prime}(E(\hat{t}, u=0))$. Therefore,

$$
W_{t}(L, t=\hat{t})=\frac{1}{C^{\prime \prime}} \int_{u^{0}}^{\bar{u}}\left[\hat{t}-D^{\prime}\left(E(\hat{t}, u=0)-\frac{D^{\prime \prime} u}{C^{\prime \prime}}\right] f(u) d u=\frac{-D^{\prime \prime}}{\left(C^{\prime \prime}\right)^{2}} \int_{u^{0}}^{\bar{u}} u f(u) d u\right.
$$

Under a hybrid policy, $u^{0}>\underline{u}$. Since the expectation of $u$ is equal to zero, $u^{0}>\underline{u}$ implies

$\int_{u^{0}}^{\bar{u}} u f(u) d u>0$. Therefore, $W_{t}(L, t=\hat{t})<0$ if and only if $D^{\prime \prime}>0$. Since $W$ is strictly convex, $W_{t}(L, t=\hat{t})<0$ implies that the optimal safety valve is higher than $\hat{t}$. However, note that $W_{t}(L, t=\hat{t})=0$ if $D^{\prime \prime}=0$. Thus, $\hat{t}$ is the optimal safety valve when expected marginal damage is constant.

To prove the final part of Proposition 2, first note that the highest possible marginal abatement cost function is $-C^{\prime}(E)+\bar{u}$. Let $\bar{t}$ be the tax that equates expected marginal damage and $-C^{\prime}(E)+\bar{u}$. Using [A.2] and [A.3],

$$
\bar{t}=D^{\prime}(E(\bar{t}, \bar{u}))=d+\frac{D^{\prime \prime}(c-\bar{t})}{C^{\prime \prime}}+\frac{D^{\prime \prime} \bar{u}}{C^{\prime \prime}}=D^{\prime}(E(\bar{t}, u=0))+\frac{D^{\prime \prime} \bar{u}}{C^{\prime \prime}} .
$$


Let us now evaluate the sign of $W_{t}(L, t)$ at $\bar{t}$. Using [A.4] and $\bar{t}-D^{\prime}(E(\bar{t}, u=0))=D^{\prime \prime} \bar{u} / C^{\prime \prime}$ from [A.5],

$$
\begin{aligned}
W_{t}(L, t=\bar{t}) & =\frac{1}{C^{\prime \prime}} \int_{u^{0}}^{\bar{u}}\left[\bar{t}-D^{\prime}\left(E(\bar{t}, u=0)-\frac{D^{\prime \prime} u}{C^{\prime \prime}}\right] f(u) d u=\frac{1}{C^{\prime \prime}} \int_{u^{0}}^{\bar{u}}\left[\frac{D^{\prime \prime} \bar{u}}{C^{\prime \prime}}-\frac{D^{\prime \prime} u}{C^{\prime \prime}}\right] f(u) d u\right. \\
& =\frac{D^{\prime \prime}}{\left(C^{\prime \prime}\right)^{2}}\left[\int_{u^{0}}^{\bar{u}} \bar{u} f(u) d u-\int_{u^{0}}^{\bar{u}} u f(u) d u\right] .
\end{aligned}
$$

Since $\int_{u^{0}}^{\bar{u}} \bar{u} f(u) d u-\int_{u^{0}}^{\bar{u}} u f(u) d u>0, W_{t}(L, t=\bar{t})>0$ as long as $D^{\prime \prime}>0$. In turn, $W_{t}(L, t=\bar{t})>0$ implies that the optimal safety valve is strictly less than $\bar{t}$. QED

Proof of Proposition 3: As noted in the text, expected emissions under the hybrid policy, conditional on the safety valve being used, are:

$$
\int_{u^{0}}^{\bar{u}} E(t, u) f(u) d u / \int_{u^{0}}^{\bar{u}} f(u) d u
$$

Substitute $E(t, u)=(c-t+u) / C^{\prime \prime}$ from [12] into [A.6] to obtain

$$
\left\{\frac{c-t}{C^{\prime \prime}} \int_{u^{0}}^{\bar{u}} f(u) d u+\frac{1}{C^{\prime \prime}} \int_{u^{0}}^{\bar{u}} u f(u) d u\right\} / \int_{u^{0}}^{\bar{u}} f(u) d u=\frac{c-t}{C^{\prime \prime}}+\frac{\int_{u^{0}}^{\bar{u}} u f(u) d u}{C^{\prime \prime} \int_{u^{0}}^{\bar{u}} f(u) d u} .
$$

We want to compare the conditional expectation of emissions to $\hat{E}=(c-d) /\left(C^{\prime \prime}+D^{\prime \prime}\right)$ (from [13]), so we want to determine the sign of

$$
\frac{c-t}{C^{\prime \prime}}+\frac{\int_{u^{0}}^{\bar{u}} u f(u) d u}{C^{\prime \prime} \int_{u^{0}}^{\bar{u}} f(u) d u}-\frac{c-d}{C^{\prime \prime}+D^{\prime \prime}}
$$

Now calculate 


$$
\frac{c-t}{C^{\prime \prime}}-\frac{c-d}{C^{\prime \prime}+D^{\prime \prime}}=\frac{c D^{\prime \prime}+d C^{\prime \prime}-t\left(C^{\prime \prime}+D^{\prime \prime}\right)}{C^{\prime \prime}\left(C^{\prime \prime}+D^{\prime \prime}\right)}
$$

Since $\hat{t}=\left(d C^{\prime \prime}+c D^{\prime \prime}\right) /\left(C^{\prime \prime}+D^{\prime \prime}\right)$ from [14],

$$
\frac{c-t}{C^{\prime \prime}}-\frac{c-d}{C^{\prime \prime}+D^{\prime \prime}}=\frac{\hat{t}-t}{C^{\prime \prime}}
$$

This value is non-positive because $t \geq \hat{t}$ from Proposition 2. Substitute [A.8] into [A.7] to rewrite [A.7] as

$$
\frac{\hat{t}-t}{C^{\prime \prime}}+\left[\int_{u^{0}}^{\bar{u}} u f(u) d u / C^{\prime \prime} \int_{u^{0}}^{\bar{u}} f(u) d u\right]
$$

which has the same sign as

$$
\hat{t}-t+\left[\int_{u^{0}}^{\bar{u}} u f(u) d u / \int_{u^{0}}^{\bar{u}} f(u) d u\right] .
$$

Thus the sign of [A.9] is the same as the sign of the expectation of emissions given the safety valve is used minus $\hat{E}$.

Now substitute [A.2] into [9] to obtain

$$
\begin{aligned}
W_{t}(L, t) & =\frac{1}{\left(C^{\prime \prime}\right)^{2}} \int_{u^{0}}^{\bar{u}}\left[t\left(C^{\prime \prime}+D^{\prime \prime}\right)-\left(d C^{\prime \prime}+c D^{\prime \prime}\right)-u D^{\prime \prime}\right] f(u) d u \\
& =\frac{t\left(C^{\prime \prime}+D^{\prime \prime}\right)-\left(d C^{\prime \prime}+c D^{\prime \prime}\right)}{\left(C^{\prime \prime}\right)^{2}} \int_{u^{0}}^{\bar{u}} f(u) d u-\frac{D^{\prime \prime}}{\left(C^{\prime \prime}\right)^{2}} \int_{u^{0}}^{\bar{u}} u f(u) d u=0 .
\end{aligned}
$$

Note that $W_{t}(L, t)=0$ if and only if

$$
t\left(C^{\prime \prime}+D^{\prime \prime}\right)-\left(d C^{\prime \prime}+c D^{\prime \prime}\right)=D^{\prime \prime} \int_{u^{0}}^{\bar{u}} u f(u) d u / \int_{u^{0}}^{\bar{u}} f(u) d u
$$

Divide [A.10] by $C^{\prime \prime}+D^{\prime \prime}$ to obtain

$$
t-\hat{t}=\frac{D^{\prime \prime}}{C^{\prime \prime}+D^{\prime \prime}} \int_{u^{0}}^{\bar{u}} u f(u) d u / \int_{u^{0}}^{\bar{u}} f(u) d u
$$


Use [A.11] to substitute for $\hat{t}-t$ in [A.9] and combine terms to rewrite [A.9] as

$$
\frac{C^{\prime \prime}}{C^{\prime \prime}+D^{\prime \prime}} \int_{u^{0}}^{\bar{u}} u f(u) d u / \int_{u^{0}}^{\bar{u}} f(u) d u>0
$$

The sign of [A.12] follows because: 1) $\left.C^{\prime \prime} /\left(C^{\prime \prime}+D^{\prime \prime}\right)>0 ; 2\right) \int_{u^{0}}^{\bar{u}} f(u) d u>0$, and 3)

$$
\int_{\underline{u}}^{\bar{u}} u f(u) d u=0 \text { and } u^{0}>\underline{u} \text { imply } \int_{u^{0}}^{\bar{u}} u f(u) d u>0 \text {. Since [A.7] has the same sign as [A.12] the }
$$

positive sign of [A.12] reveals that the expectation of emissions when the safety valve is used is strictly greater than $\hat{E}$. QED

Proof of Proposition 4: Recall that the safety valve will be used when $u>u^{0}$. Therefore, the probability that the safety valve will be used is the probability that $u$ exceeds $u^{0}$; that is,

$$
\int_{u^{0}}^{\bar{u}} f(u) d u
$$

Since the distribution of $u$ is symmetric, the probability that $u$ exceeds $u^{0}$ is less than one-half if $u^{0}>0$. The proof of Proposition 4 proceeds by evaluating $W_{t}(L, t)$ at $t$ such that $u^{0}=0$, given an optimal supply of permits $L$. If $W_{t}(L, t)<0$ the optimal $t$ is higher, $u^{0}>0$, and the probability the safety valve will be used is less than one-half. On the other hand, if $W_{t}(L, t)>0$, then the optimal $t$ is lower, $u^{0}<0$, and the probability the safety valve will be used is greater than one-half. More compactly:

$$
\operatorname{sign}\left[W_{t}(L, t)\right]=\operatorname{sign}\left[\int_{u^{0}}^{\bar{u}} f(u) d u-\frac{1}{2}\right]
$$

where $W_{t}(L, t)$ is evaluated at an optimal supply of permits and $t$ such that $u^{0}=0$. 
Recall from the proof of Proposition 2 that the first-order condition for the optimal safety valve [9] can be written as [A.4]. In turn, [A.4] can be written as

$$
W_{t}(L, t)=\frac{\left[t-D^{\prime}(E(t, u=0))\right]}{C^{\prime \prime}} \int_{u^{0}}^{\bar{u}} f(u) d u-\frac{D^{\prime \prime}}{\left(C^{\prime \prime}\right)^{2}} \int_{u^{0}}^{\bar{u}} u f(u) d u=0 .
$$

$W_{t}(L, t)$ evaluated at $u^{0}=0$ is

$$
W_{t}(L, t)=\frac{\left[t-D^{\prime}(E(t, u=0))\right]}{C^{\prime \prime}} \int_{0}^{\bar{u}} f(u) d u-\frac{D^{\prime \prime}}{\left(C^{\prime \prime}\right)^{2}} \int_{0}^{\bar{u}} u f(u) d u .
$$

Let us now determine the optimal $L$ given $u^{0}=0$. Recall from the proof of Proposition 1 that the first order condition for the optimal $L$ can be written as [A.1]. Next note from [3] that $u^{0}=0$ implies $t=-C^{\prime}(L)$. Substitute this and $u^{0}=0$ into [A.1] to obtain

$$
W_{L}(L, t)=-\left[t-D^{\prime}(L)\right] \int_{\underline{u}}^{0} f(u) d u-\int_{\underline{u}}^{0} u f(u) d u=0 .
$$

From [4], $u^{0}=0$ implies $E(t, u=0)=L$. Substitute this into [A.16] and use the symmetry of $u$ to show that [A.16] implies

$$
\left[t-D^{\prime}(E(t, u=0)] \int_{0}^{\bar{u}} f(u) d u=\int_{0}^{\bar{u}} u f(u) d u .\right.
$$

Substitute [A.17] into [A.15] to obtain

$$
W_{t}(L, t)=\frac{1}{C^{\prime \prime}} \int_{0}^{\bar{u}} u f(u) d u-\frac{D^{\prime \prime}}{\left(C^{\prime \prime}\right)^{2}} \int_{0}^{\bar{u}} u f(u) d u=\frac{\left(C^{\prime \prime}-D^{\prime \prime}\right)}{\left(C^{\prime \prime}\right)^{2}} \int_{0}^{\bar{u}} u f(u) d u .
$$

Since the expectation of $u$ is zero, $\int_{0}^{\bar{u}} u f(u) d u>0$. Thus, the sign of $W_{t}(L, t)$ at $u^{0}=0$ and an optimal supply of permits is the same as the sign of $C^{\prime \prime}-D^{\prime \prime}$. Using [A.13],

$$
\operatorname{sign}\left[C^{\prime \prime}-D^{\prime \prime}\right]=\operatorname{sign}\left[\int_{u^{0}}^{\bar{u}} f(u) d u-\frac{1}{2}\right] \text {. }
$$


[A.19] indicates that the probability the safety valve is used is less than one-half if and only if $D^{\prime \prime}>C^{\prime \prime}$. QED

Proof of Proposition 5: Given the optimal $t$ and $L$, let us define $\tilde{u}$ as $-C^{\prime}(\hat{E})+\tilde{u}=t$. This value of $u$ identifies the marginal abatement cost function that will lead firms to respond to $t$ by choosing $\hat{E}$. Aggregate emissions will be greater than $\hat{E}$ for values of $u>\tilde{u}$. (Aggregate emissions will be less than $\hat{E}$ for values of $u<\tilde{u}$ ). Moreover, since $u$ has a symmetric distribution, the probability that aggregate emissions will be greater than $\hat{E}$ is strictly less than one-half if $\tilde{u}>0$. Now $-C^{\prime}(E)=C-C^{\prime \prime} E$, [13], and [14] reveal $-C^{\prime}(\hat{E})=\hat{t}$. Then, $-C^{\prime}(\hat{E})+\tilde{u}=t$ implies $\hat{t}+\tilde{u}=t$. From Proposition 3, $t>\hat{t}$ when marginal damage is increasing. This implies $\tilde{u}>0$ and the probability that aggregate emissions will be greater than $\hat{E}$ is strictly less than one-half. If expected marginal damage is a constant, then $t=\hat{t}, \tilde{u}=0$, and the probability that emissions exceed $\hat{E}$ is equal to one-half. QED.

Proof of Proposition 6: To change the slope of $D^{\prime}(E)=d+D^{\prime \prime} E$ while forcing it to rotate around $(\hat{E}, \hat{t})$ we make $d$ a function of $D^{\prime \prime}$ and choose $d\left(D^{\prime \prime}\right)$ so that $\partial \hat{t} / \partial D^{\prime \prime}=0$. Substitute $d=d\left(D^{\prime \prime}\right)$ into [14] and differentiate the results with respect to $D^{\prime \prime}$ to obtain

$$
\frac{\partial \hat{t}}{\partial D^{\prime \prime}}=\frac{\left(d^{\prime}\left(D^{\prime \prime}\right) C^{\prime \prime}+c\right)\left(C^{\prime \prime}+D^{\prime \prime}\right)-\left(d^{\prime \prime} € c D^{\prime \prime}\right)}{\left(C^{\prime \prime}+D^{\prime \prime}\right)^{2}} .
$$

It is straightforward to show that $\partial \hat{t} / \partial D^{\prime \prime}=0$ if and only if $d^{\prime}\left(D^{\prime \prime}\right)=-(c-d) /\left(C^{\prime \prime}+D^{\prime \prime}\right)$.

Compare this to [13] to note that 


$$
d^{\prime}\left(D^{\prime \prime}\right)=-\hat{E} .
$$

We now determine the comparative statics $\partial L / \partial D^{\prime \prime}$ and $\partial t / \partial D^{\prime \prime}$. These are the solutions

to

$$
\left[\begin{array}{ll}
W_{L L} & W_{L t} \\
W_{L t} & W_{t t}
\end{array}\right]\left[\begin{array}{l}
\partial L / \partial D^{\prime \prime} \\
\partial t / \partial D^{\prime \prime}
\end{array}\right]=\left[\begin{array}{l}
-W_{L D^{\prime \prime}} \\
-W_{t D^{\prime \prime}}
\end{array}\right],
$$

where the subscripts indicate second derivatives of the expected social welfare function $W$. Let $H$ be the Hessian matrix of $W$. Strict convexity of $W$ at an optimal hybrid policy requires $W_{L L}>0$, $W_{t t}>0$, and $|H|>0$.

In addition we need the signs of $W_{L t}, W_{L D^{\prime \prime}}$, and $W_{t D^{\prime \prime}}$. Use [8] and [3] to obtain

$$
W_{L t}=-\left(t-D^{\prime}(L)\right)<0 .
$$

The sign of $W_{L t}$ follows because $L<\hat{E}$ (Proposition 1), $t>\hat{t}$ (Proposition 2), and $D^{\prime}(L)$ upward sloping implies $t>D^{\prime}(L)$.

To obtain $W_{L D^{\prime \prime}}$ first substitute $D^{\prime}(L)=d\left(D^{\prime \prime}\right)+D^{\prime \prime} L$ into [8] to obtain

$$
W_{L}=\int_{\underline{u}}^{u^{0}}\left[C^{\prime}(L)-u+d\left(D^{\prime \prime}\right)+D^{\prime \prime} L\right] f(u) d u=0 .
$$

Differentiate this with respect to $D^{\prime \prime}$ and substitute [A.20] into the result to obtain

$$
W_{L D^{\prime \prime}}=\int_{\underline{u}}^{u^{0}}(L-\hat{E}) f(u) d u<0 .
$$

The sign of [A.23] follows because $L<\hat{E}$ (Proposition 1).

To obtain $W_{t D^{\prime \prime}}$, first substitute $D^{\prime}(E(t, u))=d\left(D^{\prime \prime}\right)+D^{\prime \prime} E(t, u)$ into [9] to obtain

$$
W_{t}=\frac{1}{C^{\prime \prime}} \int_{u^{0}}^{\bar{u}}\left[t-d\left(D^{\prime \prime}\right)-D^{\prime \prime} E(t, u)\right] f(u) d u=0 .
$$

Differentiate this with respect to $D^{\prime \prime}$ to obtain 


$$
W_{t D^{\prime \prime}}=\frac{1}{C^{\prime \prime}} \int_{u^{0}}^{\bar{u}}[\hat{E}-E(t, u)] f(u) d u,
$$

Which can be rewritten as

$$
W_{t D^{\prime \prime}}=\frac{1}{C^{\prime \prime}} \int_{u^{0}}^{\bar{u}} f(u) d u\left\{\hat{E}-\frac{\int_{u^{0}}^{\bar{u}} E(t, u) f(u) d u}{\int_{u^{0}}^{\bar{u}} f(u) d u}\right\}<0 .
$$

The sign of [A.24 follows in part because the first term in the product is positive. Moreover, the term in brackets is the difference between $\hat{E}$ and expected emissions given the safety valve is used, which is negative according to Proposition 3.

We now have enough information to determine the signs of $\partial L / \partial D^{\prime \prime}$ and $\partial t / \partial D^{\prime \prime}$. From [A.21]:

$$
\begin{aligned}
& \partial L / \partial D^{\prime \prime}=\left[-W_{L D^{\prime \prime}} W_{t t}+W_{t D^{\prime \prime}} W_{L t}\right] /|H|>0 ; \\
& \partial t / \partial D^{\prime \prime}=\left[-W_{L L} W_{t D^{\prime \prime}}+W_{L D^{\prime \prime}} W_{L t}\right] /|H|>0 .
\end{aligned}
$$

The positive signs of $\partial L / \partial D^{\prime \prime}$ and $\partial t / \partial D^{\prime \prime}$ follow because $W_{L L}>0, W_{t t}>0,|H|>0, W_{L t}<0$ ([A.22]), $W_{L D^{\prime \prime}}<0([\mathrm{~A} .23])$, and $W_{t D^{\prime \prime}}<0$ ([A.24]). This proves part 1) of the Proposition.

To prove part 2) of the Proposition, note first that the probability the safety valve will be used is the probability that $u \geq u^{0}$, where recall (from [3]) that $u^{0}=t+C^{\prime}(L)$. Differentiate

$$
\int_{u^{0}}^{\bar{u}} f(u) d u, u^{0}=t\left(D^{\prime \prime}\right)+C^{\prime}\left(L\left(D^{\prime \prime}\right)\right)
$$

with respect to $D^{\prime \prime}$ to obtain

$$
-\left\{\frac{\partial t}{\partial D^{\prime \prime}}+C^{\prime \prime} \frac{\partial L}{\partial D^{\prime \prime}}\right\} f\left(u^{0}\right)<0
$$


The sign of this expressions follows because $C^{\prime \prime}>0$, and $\partial t / \partial D^{\prime \prime}>0$ and $\partial L / \partial D^{\prime \prime}>0$ from part 1) of the Proposition. The sign indicates that the probability that the safety valve is used is decreasing in the steepness of the expected marginal damage function.

For part 3) of the Proposition, recall that expected aggregate emissions under a hybrid policy are

$$
L \int_{\bar{u}}^{u^{0}} f(u) d u+\int_{u^{0}}^{\bar{u}} E(t, u) f(u) d u .
$$

Substitute $t=t\left(D^{\prime \prime}\right)$ and $L=L\left(D^{\prime \prime}\right)$ into [A.25], noting that $E\left(t\left(D^{\prime \prime}\right), u\right)=\left(c-t\left(D^{\prime \prime}\right)+u\right) / C^{\prime \prime}$ from [12] and, again, $u^{0}=t\left(D^{\prime \prime}\right)+C^{\prime}\left(L\left(D^{\prime \prime}\right)\right)$. Differentiate the result with respect to $D^{\prime \prime}$ to obtain

$$
\left(L-E\left(t, u^{0}\right)\right) f\left(u^{0}\right) \frac{\partial u^{0}}{\partial D^{\prime \prime}}-\int_{u^{0}}^{\bar{u}} \frac{1}{C^{\prime \prime}} \frac{\partial t}{\partial D^{\prime \prime}} f(u) d u
$$

The first term is equal to zero because $E\left(t, u^{0}\right)=L$ from [4]. Thus, the effect of $D^{\prime \prime}$ on expected aggregate emissions is

$$
-\int_{u^{0}}^{\bar{u}} \frac{1}{C^{\prime \prime}} \frac{\partial t}{\partial D^{\prime \prime}} f(u) d u<0,
$$

the sign of which follows because $C^{\prime \prime}>0$ and $\partial t / \partial D^{\prime \prime}>0$. That this term is negative implies that expected aggregate emissions are decreasing in the steepness of the expected marginal damage function. QED 


\section{References}

Aldy, Joseph E., Scott Barrett, Robert N. Stavins. 2003. "Thirteen plus one: a comparison of global climate policy architectures.” Climate Policy 3(4), 373-397.

Burtraw, Dallas, Karen Palmer, and Danny Kahn. 2009. “A symmetric safety valve.” Resources for the Future Discussion Paper, RFF DP 09-06.

Holdren, John P., et al. 2004. Ending the Energy Stalemate: A Bipartisan Strategy to Meet America's Energy Challenges. National Commission on Energy Policy. Washington, DC.

Jacoby, Henry D. and A. Denny Ellerman. 2004. “The safety valve and climate policy.” Energy Policy 32(4), 481-491.

McKibbin, Warwick J., and Peter J. Wilcoxen. 1997. "A better way to slow global climate change.” Policy Brief No. 17. Brookings Institution, Washington, D.C.

McKibbin, Warwick J., and Peter J. Wilcoxen. 2002. "The role of economics in climate change policy.” Journal of Economics Perspectives 16(2), 107-129.

Murray, Brian C., Richard G. Newell and William A. Pizer. 2009. "Balancing cost and emissions certainty: An allowance reserve for cap-and-trade.” Review of Environmental Economics and Policy 3(1), 84-103.

Philibert, Cédric. 2008. "Price caps and price floors in climate policy: A quantitative assessment.” International Energy Agency Information Paper. OECD/IEA.

Pizer, William A. 2002. “Combining price and quantity controls to mitigate global climate change.” Journal of Public Economics 85(3), 409-434.

Roberts, M.J. and M. Spence. 1976. "Effluent charges and licenses under uncertainty," Journal of Public Economics 5(3-4), 193-208.

Samuelsohn, Darren. 2008. Behind "safety valve” debate resides 30+ years of history. Climate Wire, March 11.

Stavins, Robert N. 2008. “Addressing climate change with a comprehensive US cap-and-trade system.” Oxford Review of Economic Policy 24(2), 298-321.

Weitzman, Martin. 1974. "Prices vs. quantities," Review of Economic Studies 41(4), 477-91. 